\title{
A Rare Retrograde Intrarenal Surgery Complication: Hemophagocytic Syndrome
}

\author{
Murat Akgül, MD, ${ }^{1}$ Cenk Yazıcı, MD, ${ }^{1}$ Hüseyin Ateş, MD, ${ }^{1}$ Enes Altın, MD, ${ }^{1}$ and Burhan Turgut, MD ${ }^{2}$
}

\begin{abstract}
Background: Hemophagocytic syndrome (HS) is a syndromic complex that is categorized in the group of histiocytic disorders associated with macrophages.

Case Presentation: A 39-year-old male patient was admitted to the outpatient clinic with complaint of left flank pain. A $1 \mathrm{~cm}$ kidney stone was found in the upper pole of left kidney at radiologic imaging. The patient underwent retrograde intrarenal surgery (RIRS) with no peroperative complication. High fever and increasing of acute-phase reactants were observed at postoperative first day. Besides resistant fever, pancytopenia developed despite the appropriate antibiotherapy. The urine and blood cultures were sterile. After multidisciplinary consultation, bone marrow sampling was performed. Microscopic examination of the bone marrow material revealed that the macrophage cells phagocyted the erythrocytes, which was compatible with HS. Unfortunately despite the appropriate medical HS treatment, the patient died due to multiorgan failure at the 21st day of RIRS.

Conclusion: HS is a significantly rare complication after RIRS, which was presented initially with postoperative fever. HS should be kept in mind if the patient had resistant fever and pancytopenia despite the appropriate antibiotherapy.
\end{abstract}

Keywords: retrograde intrarenal surgery, hemophagocytic syndrome, complication

\section{Introduction and Background}

$\mathbf{H}$ EMOPHAGOCYTIC SYNDROME (HS) is a syndromic complex that is categorized in the group of histiocytic disorders associated with macrophages. HS represents not a single disease, but a clinical syndrome in which different conditions form the same type of inflammatory response phenotype. Activation of macrophages and $\mathrm{T}$ lymphocytes causes severe inflammation and multiple organ failure. HS is characterized by fever, hepatosplenomegaly, and cytopenia as a result of overproduction of proinflammatory cytokines and hemophagocytosis. HS is divided into two different groups; genetic and acquired. ${ }^{1}$ Surgical stress and infection may cause HS. In this report, we presented a case of HS with fever, progressive pancytopenia, and impaired liver function that occurred after the retrograde intrarenal surgery (RIRS).

\section{Presentation of Case}

A 39-year-old male patient without any comorbidity applied to the urology clinic with the complaint of left flank pain. In the noncontrast-enhanced CT, 10 and $3 \mathrm{~mm}$ stones were detected in the upper pole of the left kidney (Fig. 1). The urine culture was sterile before the operation. The patient underwent RIRS with an operation time of 45 minutes.

A prophylactic single-dose ceftriaxone $1000 \mathrm{mg}$ was applied 1 hour before the operation. The surgery started with urethrocystoscopy and insertion of a guidewire to the ipsilateral kidney. According to our surgical technique, we performed $8 \mathrm{~F}$ semirigid ureteroscopy to the ipsilateral ureter just before the insertion of access sheath. By the help of the semirigid ureteroscope, we visualized the ureter till to the renal pelvis and performed active ureteral dilation for insertion of ureteral access sheath. Just after the semirigid ureteroscopy, we introduced an $11 \mathrm{~F}-13 \mathrm{~F}$ ureteral access sheath over the guidewire and located it $1 \mathrm{~cm}$ below the ureteropelvic junction under fluoroscopic view. A digital flexible ureteroscope (Karl-Storz Flex $\mathrm{X}^{\mathrm{c}}$ ) was inserted to the kidney through the access sheath, and stone fragmentation was performed by reusable $270 \mathrm{~nm}$ fiber and holmium laser (Quanta System ${ }^{\mathrm{TM}}$ ). At the end of the operation, a 4.7F. Double-J stent was introduced to the renal system. There was no peroperative complication during surgery. After the RIRS, the stone-free status was provided.

At the postoperative first day of surgery, the patient had $39^{\circ} \mathrm{C}$ fever. As the C-reactive protein (CRP) and procalcitonin levels $(200 \mathrm{mg} / \mathrm{L}$ and $4.11 \mathrm{ng} / \mathrm{mL}$, respectively) were

\footnotetext{
${ }^{1}$ Urology Department and ${ }^{2}$ Hematology Department, Faculty of Medicine, Tekirdağ Namık Kemal University, Tekirdağ, Turkey.
} 


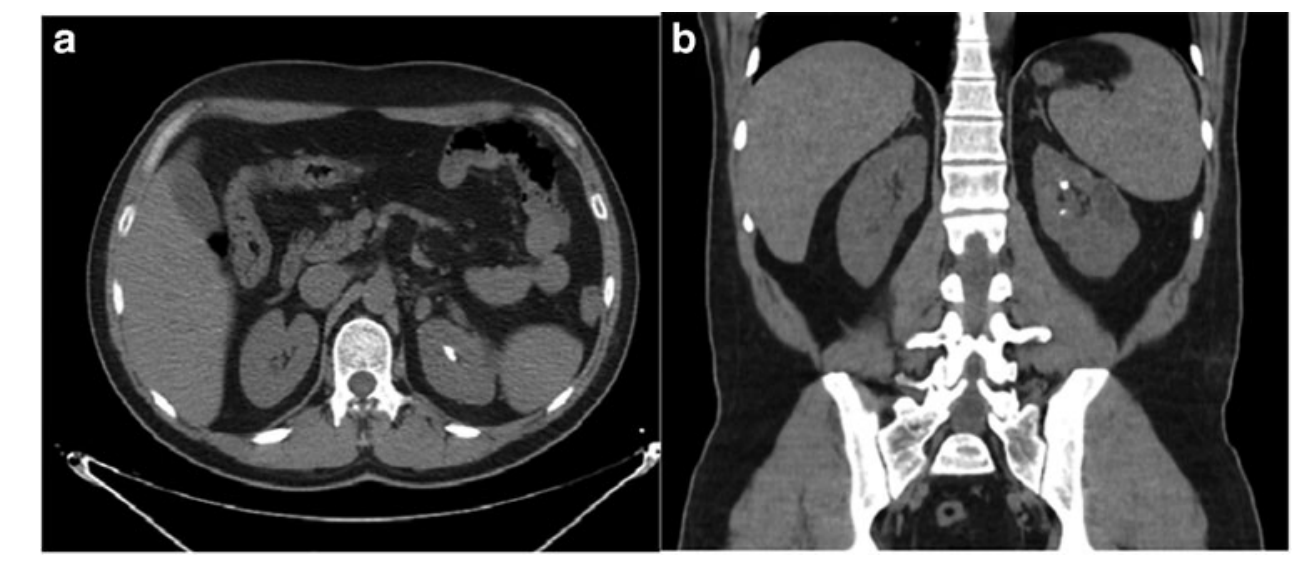

FIG. 1. Noncontrast abdominopelvic CT image. (a) Transverse section and (b) coronal section.

high, the patient was consulted to the infectious disease department. Urine and blood cultures were taken before antibiotic application. Piperacillin/tazobactam $4.5 \mathrm{~g} / \mathrm{qid}$ was administered empirically to the patient. On the second day of antibiotic treatment, pancytopenia was developed (hemoglobin: $11.3 \mathrm{~g} / \mathrm{dL}$, white blood cell: 1.63 , platelet: 77,000$)$ and the fever persisted. In the early postoperative days, anemia was not as severe as leucopenia and thrombocytopenia. However, anemia also developed a few days later and multiple blood transfusions were required. As the patient was resistant to piperacillin/tazobactam treatment, the antibiotherapy was changed to meropenem $1 \mathrm{~g} / \mathrm{tid}$. Both the urine and blood cultures were repetitively negative. However, the fever persisted despite the antibiotherapy. At the radiologic evaluation, we observed hepatosplenomegaly and there was no intra-abdominal abscess formation. The biochemical analyses on postoperative fourth day were as follows: total bilirubin: $4.76 \mathrm{mg} / \mathrm{dL}$, direct bilirubin: $4.24 \mathrm{mg} / \mathrm{dL}$, Aspartate aminotransferase (AST): 395 IU/L, Alanine aminotransferase (ALT): 200 IU/L, Gamma-glutamyl transferase (GGT): 236 IU/L, Lactate dehydrogenase (LDH): 774 IU/L, Alkaline phosphatase (ALP): $140 \mathrm{IU} / \mathrm{L}, \mathrm{CRP}: 97 \mathrm{mg} / \mathrm{L}$, and procalcitonin: $2.31 \mathrm{ng} / \mathrm{mL}$. With this biochemical evaluation, the patient was consulted to the hematology, infectious disease, and gastroenterology clinics. To cover possible fungal infection, fluconazole was added to his treatment, but the expected improvement was not observed.

At the postoperative seventh day, the patient had pancytopenia, hypofibrinogenemia with high ferritin levels. To evaluate the reason of the pancytopenia, peripheral blood smear was analyzed by the hematologists. According to the results of peripheral blood smear, hematologists performed a bone marrow biopsy at the postoperative seventh day. An increase in the granulocyte series was seen in the bone marrow material. Microscopic examination of the bone marrow revealed the macrophage cells that phagocyted the erythrocytes. This finding was compatible with HS (Fig. 2). With the diagnosis of HS, dexamethasone and etoposide treatment was initiated. At the end of the 3 week of treatment, the patient died due to multiorgan failure.

\section{Discussion and Literature Review}

HS is defined as a benign histiocytic proliferation associated with viral infections. Several etiologic factors such as genetic factors, bacterial infections, tissue trauma, and tumors were found to be related with HS (Table 1). ${ }^{1}$ Macrophages, which are the main cellular component of the immune system, were responsible for this hazardous disease. HS represents not a single disease, but a clinical syndrome in which different conditions produce the same type of inflammatory response phenotype. It is divided into two different groups: genetic and acquired (Table 1). While the genetic types of HS have been observed in children and the young adult population, acquired HS can be present in all age groups. ${ }^{1,2}$

Genetic HS is an inherited autosomal recessive disorder. The presence of familial history or consanguineous marriages are the main indicator to diagnose familial HS. If there is no diagnosed case or consanguineous marriage in the family, it is considered to be sporadic HS. In a Swedish study, the incidence of HS for children younger than 15 years was reported to be $1.2 / 10,00,000$ cases per year. Most of the cases $(70 \%$ $80 \%$ ) are diagnosed at first age. Generally, the patients have no symptoms at birth and it takes some time for the symptoms to become apparent. There are an extremely limited number of cases that were reported in the in utero period or immediately

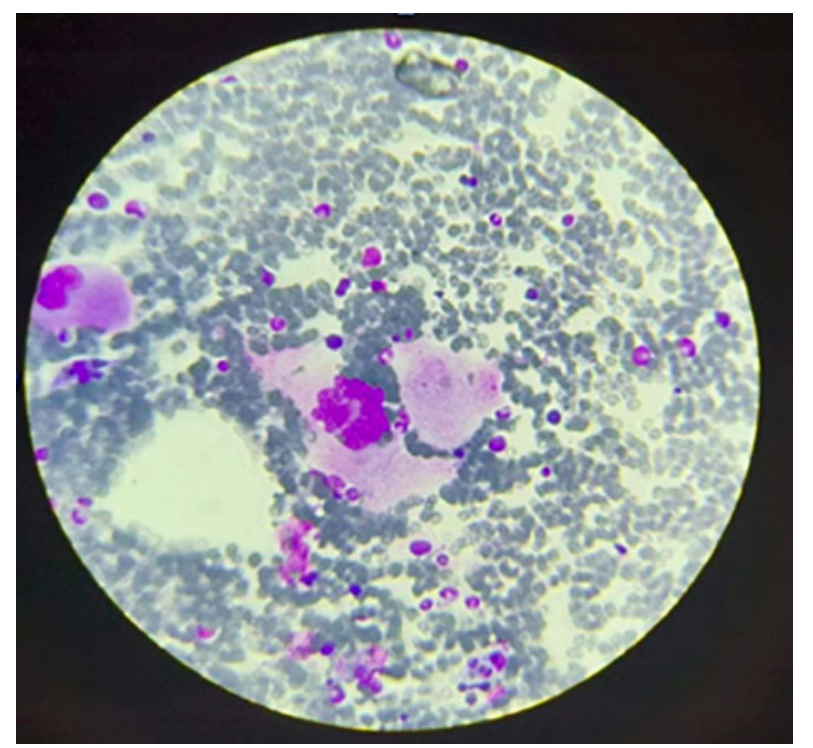

FIG. 2. Microscopic examination of the bone marrow material revealed that the macrophage cells phagocyted the erythrocytes. 
TABle 1. The Classification

of Hemophagocytic Syndrome

\section{Genetic (primary) HS}

Familial HS (Farquhar disease)

Immune deficiency syndrome

CHS 1

GS 2

XLP

Acquired (secondary) HS

Exogen agents (infection, toxins)

Endogen factors (tissue damage, metabolic product, radical stress)

Rheumatic diseases

Tumors

CHS 1, Chediak-Higashi syndrome 1; GS 2, Griscelli syndrome 2; HS, hemophagocytic syndrome; XLP, X-linked lymphoproliferative syndrome.

after birth. Most of the patients were diagnosed late in the adolescent or adult period. ${ }^{3,4}$ Having an infection may be a triggering factor in the development of primary HS. EpsteinBarr virus (EBV) is the most common viral factor, which infects B cells, T cells, and natural killer (NK) cells and leads to monoclonal or oligoclonal proliferation of these cells. Other viruses such as the herpes group viruses, cytomegalovirus, varicella zoster virus, human herpes virus 6-8, can also cause HS. ${ }^{5,6}$ In addition to viral factors, bacterial, protozoal, fungal, parasitic diseases, malignancies, radical stress, metabolic diseases, immune deficiency, and collagen tissue disease may also lead to HS. ${ }^{1}$

The clinical manifestations of HS occur with phagocytosis of leukocytes, erythrocytes, and platelets as a result of monocyte, macrophage, and histiocyte activation, and proliferation in tissues such as bone marrow, liver, spleen, lymph node, and skin. The main accepted opinion on HS pathophysiology is the cytokine storm caused by insufficient apoptosis of NK cells due to genetic defects and proliferation of activated $\mathrm{T}$ cells with macrophage activation. As a result, hemophagocytosis, cytopenia, infection, fever, and bleeding develop. This condition cannot be easily separated from sepsis and disseminated intravascular coagulation. ${ }^{2}$ There are diagnostic criteria set by the "Histiocyte Society" to define HS (Table 2). According to this definition, HS is diagnosed if the patient met five of the eight criteria. ${ }^{7,8}$

The most typical findings of HS are fever, hepatosplenomegaly, and cytopenia. The body temperature of the patients is above $38.5^{\circ} \mathrm{C}$, which lasts longer than 7 days. Ecchymosis and pallor secondary to pancytopenia can also be seen. In some cases, icterus may occur due to hepatic and hematopoietic dysfunction. Nonspecific rash can also be seen in $65 \%$ of HS patients. Neurologic symptoms such as convulsions, ataxia, hemiplegia, mental disorders, and irritability have been reported. Fatigue, loss of appetite, and weight loss can also be seen. ${ }^{2}$

Whether the diagnosis is genetic or acquired HS, the primary aim of the treatment is to block the increased cytokine cascade and suppress hyperinflammation. The secondary aim is to kill antigen-presenting cells and eliminate the ongoing inflammatory response. The main components of treatment are dexamethasone, etoposide, cyclosporine, and intravenous immunoglobulin. Corticosteroids act by suppressing excess cytokines. It is also an advantage that they can cross the
Table 2. Hemophagocytic Syndrome DiAgNOSIS CRITERIA

\section{Clinical criteria}

Fever: More than $38^{\circ} \mathrm{C}$ fever for 7 days or more

Splenomegaly: Palpable or more than $3 \mathrm{~cm}$

Laboratory criteria

Cytopenia:

Hemoglobin $<9 \mathrm{~g} / \mathrm{dL}$

Platelets $<100,000 / \mathrm{mm}^{3}$

Absolute neutrophil count $<1000 / \mathrm{mm}^{3}$

Hypertriglyceridemia and/or hypofibrinogenemia

Triglyceridemia $>265 \mathrm{mg} / \mathrm{dL}$

Fibrinogen $<1.5 \mathrm{~g} / \mathrm{L}$

Ferritin $>500 \mu \mathrm{g} / \mathrm{L}$

Low or absent NK cell activity

Soluble CD25 > 2400 U/mL

Histopathologic criteria

Hemophagocytosis detected without malignancy in bone marrow, spleen, or lymph node

NK, natural killer.

blood/brain barrier and reach the central nervous system and suppress inflammation. Etoposide is a cytotoxic drug that targets macrophages. It also prevents cellular proliferation by preventing cell division. ${ }^{9}$

In patients with less severe symptoms, corticosteroids and immunoglobulin infusions are sometimes sufficient. However, if symptoms progress, the treating physician clinicians should not hesitate to use therapy with dexamethasone, etoposide, and cyclosporine. Etoposide may be life saving, especially in patients with HS due to EBV infection. The mortality rate was 14 times higher for patients with EBVassociated hemophagocytic lymphohistiocytosis who did not receive etoposide within the first 4 weeks. ${ }^{10}$ Prognosis is poor, depending on the associated disease, and the early mortality rate (i.e., within 1 month after diagnosis) was $20 \%$ with an overall mortality rate of $50 \% .^{11,12}$

We also observed similar clinical findings in our patient. The fever persisted after the operation despite the multiple antibiotherapies. All the urine and blood cultures were sterile. Hepatosplenomegaly was detected during physical examination and radiologic evaluation. In laboratory tests, pancytopenia, hypofibrinogenemia, hypertriglyceridemia, and increased ferritin levels were observed, and the histopathologic appearance of bone marrow biopsy was compatible with HS. By these findings, six of the eight HS diagnosing criteria were met by our patient. Initially, we started the treatment with the diagnosis sepsis. However, both the urine and blood cultures were repetitively negative and the fever persisted despite the antibiotherapy. Eventually, we performed bone marrow analysis. We had observed that the erythrocytes were phagocyted by macrophage cells in bone barrow material. In addition to this, other HS parameters accompanied the clinic of the patient. The other relevant medical branches such as hematology, infectious disease, gastroenterology, and internal medicine specialists agreed on HS. The patient and the first-degree relatives were evaluated regarding the genetic basis. However, there was no history related to HS. The patient was accepted as acquired HS secondary to the RIRS. We started with intravenous dexamethasone $8 \mathrm{~g} / \mathrm{bid}$ as the first line treatment. Two days after the first steroid treatment, 
etoposide $150 \mathrm{mg}$ was initiated for 2 days a week. However, the patient did not respond to any treatment and he died as a result of multiorgan failure at the third week of the treatment.

\section{Conclusion}

In recent years, the practice of RIRS is increasing day by day for the treatment of upper urinary tract stones all around the world. Postoperative fever is one of the prevalent complications of this surgery and mostly related with infection. On the contrary, we observed a significantly rare complication in our patient, which also caused postoperative fever. Clinicians must be aware of this rare complication after any surgery. HS caused unexpected death of a patient after a classical RIRS. Early diagnosis of HS may positively affect the fatal course of the disease.

\section{Disclosure Statement}

No competing financial interest exists.

\section{Funding Information}

No funding was received for this article.

\section{References}

1. Janka GE. Familial and acquired hemophagocytic lymphohistiocytosis. Eur J Pediatr 2007;166:95-109.

2. McClain KL, Allen CE, Hicks MJ. Histiocytic diseases. In: Pizzo PA, Poplack DG, eds., 7th edition. Principles and Practice of Pediatric Oncology. Philadelphia: Lippincott Williams \& Wilkins Co., 2015, pp. 617-627.

3. Janka GE. Hemophagocytic syndromes. Blood Rev 2007; 21:245-253.

4. Devecioğlu Ö, Sarıbeyoğlu E. Hemophagocytic lymphohistiocytosis in children. J Pediatr Sci 2009;5:64-71.

5. Henter JI, Ehrnst A, Andersson J, et al. Familial hemophagocytic lymphohistiocytosis and viral infections. Acta Paediatr 1993;82:369-372.

6. Bouffandeau B, Mofredj A, Blanc S. Hemophagocytic syndrome in the critically ill. Intensive Care Med 2001;27:948.

7. George MR. Hemophagocytic lymphohistiocytosis: Review of eitologies and management. J Blood Med 2014;5:69-86.

8. Henter JI, Elinder G, Ost A. Diagnostic guidelines for hemophagocytic lymphohistiocytosis. The FHL Study Group of the Histiocyte Society. Semin Oncol 1991;18:29-33.

9. Freeman HR, Ramanan AV. Review of haemophagocytic lymphohistiocytosis. Arch Dis Child 2011;96:688-693.
10. Imashuku $\mathrm{S}$, Kuriyama $\mathrm{K}$, Teramura $\mathrm{T}$, et al. Requirement for etoposide in the treatment of Epstein-Barr virus-associated hemophagocytic lymphohistiocytosis. J Clin Oncol 2001;19: 2665-2673.

11. Karras A, Hermine O. Hemophagocytic syndrome. La Revue Méd Intern 2002;23:768-778.

12. Rivière S, Galicier L, Coppo $\mathrm{P}$, et al. Reactive hemophagocytic syndrome in adults: A retrospective analysis of 162 patients. Am J Med 2014;127:1118-1125.

Address correspondence to: Murat Akgül, MD Urology Department Faculty of Medicine

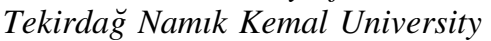
Süleymanpaşa Tekirda 59020 Turkey

E-mail: drmuratakgul@gmail.com

$\begin{aligned} & \quad \text { Abbreviations Used } \\ & \text { ALP }=\text { Alkaline phosphatase } \\ & \mathrm{ALT}=\text { Alanine aminotransferase } \\ & \mathrm{AST}=\text { Aspartate aminotransferase } \\ & \mathrm{CHS} 1=\text { Chediak-Higashi syndrome } 1 \\ & \mathrm{CRP}=\text { C-reactive protein } \\ & \mathrm{CT}=\text { computed tomography } \\ & \mathrm{EBV}=\text { Epstein-Barr virus } \\ & \mathrm{GS} 2=\text { Griscelli syndrome } 2 \\ & \mathrm{HS}=\text { hemophagocytic syndrome } \\ & \mathrm{LDH}=\text { Lactate dehydrogenase } \\ & \mathrm{NK}=\text { natural killer } \\ & \mathrm{RIRS}=\text { retrograde intrarenal surgery } \\ & \mathrm{XLP}=\text { X-linked lymphoproliferative syndrome } \\ &\end{aligned}$

Cite this article as: Akgül M, Yazıcı C, Ateş H, Altın E, Turgut B (2020) A rare retrograde intrarenal surgery complication: hemophagocytic syndrome, Journal of Endourology Case Reports 6:4, 339-342, DOI: 10.1089/ cren.2020.0066. 